\title{
Influence of grain boundaries on short fatigue crack growth in "polycrystalline CMSX-4"
}

\author{
Michael Marx ${ }^{1, a}$, Wolfgang Schäf $f^{1, b}$ and Horst Vehoff ${ }^{1, c}$ \\ ${ }^{1}$ Saarland University, Department of Materials Science and Engineering, \\ Campus D22, 66123 Saarbrücken, Germany \\ am.marx@matsci.uni-sb.de, bw.schaef@matsci.uni-sb.de, 'vehoff@matsci.uni-sb.de
}

Key words: Microstructural crack propagation, grain boundaries, focused ion beam, crack tomography

\begin{abstract}
Increasing the resistance of a material to fatigue crack growth by optimizing the microstructure is a major task of materials science. In this regard, grain boundaries and precipitates are well known to decelerate short cracks. Thereby the strength of the interaction is influenced by the crack parameters crack length and distance to the obstacles, the grain boundary parameters like orientation of the adjacent grains and the precipitate parameters like size and distance. A comprehensive understanding of the underlying physical principles is missing. The focused ion beam (FIB) microscope offers new possibilities for systematic experiments and three dimensional investigations to quantify the microstructural impact. The ion beam is used to cut micro-notches as initiation sites for cracks. Contrary to natural cracks the influencing parameters can be varied independently for a systematic investigation of the mechanisms. Additionally, the ion beam is used to make a 3D image of the crack path and the surrounding microstructural elements. The commonly single crystalline nickel base superalloy CMSX-4 served as a model material in a polycrystalline modification. Thereby it was possible for the first time to reveal quantitative data of the effect of microstructural barriers on short fatigue crack growth.
\end{abstract}

\section{Introduction}

The fatigue problem is technically divided in two different demands: Calculating the lifetime of a component and improving the fatigue resistance of a material. So far, the lifetime calculation is based on the approved techniques which use Wöhler diagrams for high cycle fatigue or calculate the lifetime by the Paris law for low cycle fatigue. For every material fatigue parameters like the constants of the Paris law and Wöhler diagrams are needed. On the other hand, the knowledge of the fatigue mechanisms would be helpful to develop materials with a higher fatigue resistance to reduce excess charges and to prolongate inspection intervals. Therefore it is necessary to know the mechanisms of the crack propagation.

First the well known Paris law describes the crack propagation rate da/dN as a function of the cyclic stress intensity factor $\Delta \mathrm{K}$ as given in equation 1 :

$$
\frac{d a}{d N}=D \cdot \Delta K^{m}
$$

However, if one takes the dislocation emission and the resulting crack tip sliding displacement into account, for stage I cracks the Paris law can be written in the following form:

$$
\frac{d a}{d N}=C \cdot \Delta C T S D^{n}
$$

Thereby the constants $\mathrm{D} / \mathrm{C}$ and $\mathrm{m} / \mathrm{n}$ are material-specific fatigue values and have to be measured for every material. This can be done by observing artificial micro cracks which can be initiated with reproducible crack length, depth or orientation. Thereby a lot of additional influences on the results like geometric crack parameters or microstructure parameters can be excluded. As it will be shown in the experimental part of the paper, for instance in large grains or single crystals cracks can be initiated which propagate in stage I by activating only the slip system with the highest Schmid factor. In this case the constants of the Paris law can be measured easily on the basis of the same boundary conditions for different materials. 
If one is interested in improving the fatigue resistance, it is important to keep in mind, that microstructural barriers influence the crack propagation rate. Especially grain boundaries reduce the crack growth sometimes even to a total stop. The models that describe the interaction of cracks with grain boundaries are based on the Bilby-Cotrell-Swinden-theory [1] model for cracks in the modification of Tanaka [2] and Navarro and De Los Rios [3] for their interaction with grain boundaries, where dislocations emitted from the crack tip are blocked at the grain boundary. Precipitates interact also with the crack dislocations whereby the precipitates sometimes are cut and sometimes have to be bypassed. Both mechanisms need a higher driving force and reduce the crack propagation. However, it has not been checked systematically what determines the strength of a local barrier against crack propagation. Therefore new techniques had to be developed to perform such systematic experiments where single parameters can be changed independently. So far this could only be investigated by simulation methods [4], but now it can be checked experimentally by the FIB tomography which gives a 3D insight in the passing process.

\section{Experimental and results}

The advantages of artificial crack initiation. The FIB is the only tool to cut reproducible micronotches with notch parameters with an accuracy of better than $1 \mu \mathrm{m}$ directly on the preferred slip system to initiate stage I cracks. In several experimental investigations there have been a lot of materials tested. We proved the FIB crack initiation for nickel based superalloys, steels and aluminium alloys. For all these materials the material parameters for the Paris law could be determined. Before the artificial notch was cut, the orientation of every grain of the coarse grained materials was measured by electron back scatter diffraction (EBSD, Fig. 1). Then the Schmid factors of all slip-systems of every grain could be calculated. Finally the grain with the highest Schmid factor was selected for crack initiation. The crack was then cut directly parallel to the preferred slip system (Fig.2a).

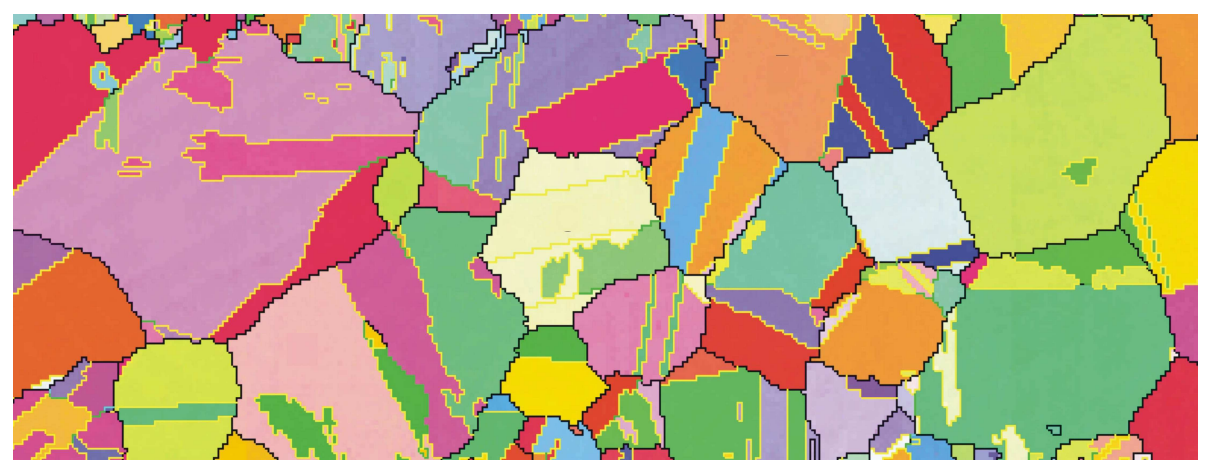

Fig.1: EBSD-grain orientation map of a polycrystalline nickel specimen. Twin boundaries are marked in yellow.

Now the crack parameters which influence the crack propagation can be varied independently. These parameters are: crack length, distance between crack tip and obstacle and in the case of grain boundaries the misorientation of the adjacent grains. Subsequently, there are two possibilities: the crack parameters can be kept constant and grain boundary parameters can be changed by selecting different grain boundaries. Or the notch parameters length and distance between initial notch and grain boundary can be changed systematically on the same grain boundary (Fig.2). This should result in a difference in the driving force of the crack at the point when the crack tip approaches the grain boundary. Figure $2 \mathrm{~b}$ shows an artificial initiated crack passing a grain boundary.

As mentioned above, this technique has been used for a lot of materials; however the nickel based superalloys have been of special interest. They are often used in the directionally solidified configuration and are a very sophisticated model material because they have long grain boundaries where several cracks with different crack parameters can be introduced near one grain boundary. 
The best specimen configuration in this case would be a bicrystal which as described in [5].

Afterwards the specimens with a $3 \times 3 \mathrm{~mm}$ cross section and a length of $45 \mathrm{~mm}$ were cycled in a Schenk servo-hydraulic testing machine at room temperature with a frequency of $5 \mathrm{~Hz}$. The load amplitude was $300 \mathrm{MPa}$ with an $R$-ratio of $R=\sigma_{\min } / \sigma_{\max }=-0.1$. The crack length and thereby the crack growth rate was measured by replica technique for every 1000 cycles. Details can be found in [6].
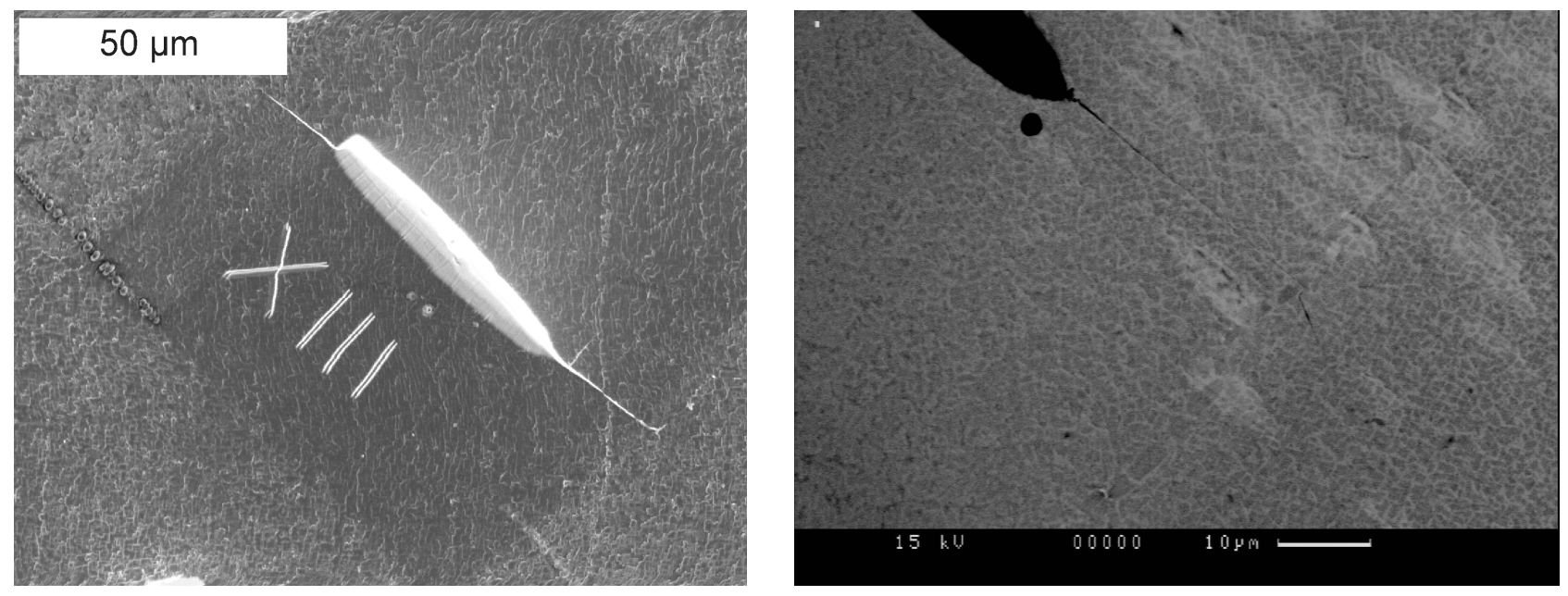

Fig.2 a) FIB notch initiated directly on the preferred (111)-slip plane calculated from the EBSD data.

b) FIB notch in front of a grain boundary with a crack emitted from the notch tip passing the grain boundary.

Crack propagation and interaction with and without obstacles. First, the parameters $\mathrm{C}$ and $\mathrm{n}$ of the Paris law (Eq.2) should be determined empirically for the CMSX4 in the used modification described above by measuring the crack growth rate and fitting the $\mathrm{da} / \mathrm{dN}$ curve. $\mathrm{C}$ is a measure for the reversibility of the dislocation emission process at the crack tip and the parameter $\mathrm{n}$ (no dimension, between 1 and 2) describes the plastic crack opening at the tip which is a combination of different crack opening modes I, II or III. All specimens were cut from the same material. Therefore the material parameter $\mathrm{C}$ can be taken as equal for all specimens. Initiating the cracks with the FIB directly on the slip planes with the highest Schmid factor (between 0,42 and 0,5) should result in a more or less simple crack opening mode II or mode III depending on the orientation of the grain. Therefore the exponent $\mathrm{n}$ is supposed to be constant for all measurements done in this experiment.

The crack propagation rate was measured during fatigue tests as were the crack length and the slip band length in front of the crack tip. For details how to measure the slip band length see [7]. From the crack length and slip band length the $\triangle$ CTSD was calculated. The relation between da/dN and $\triangle \mathrm{CTSD}$ is shown in figure 3. Using this diagram, $\mathrm{C}$ and $\mathrm{n}$ can be calculated by fitting the crack growth data according to Eq.2. These results have been revealed through several cracks in CMSX-4 with large single crystal like grains (SX1 with two cracks) and for cracks which were far away from the nearest grain boundary (DS1 and DS2 with one crack and DS3 with two cracks) respectively. C and $n$ could be determined to $C=6,7$ and $n=1,6$.

Afterwards several samples of the polycrystaline CMSX-4 have been tested to quantify the influence of grain boundaries on crack growth. For example the overall crack length in respect to the number of load cycles is shown in figure 4a. Additionally, in this figure the estimated crack length without the grain boundary ahead of the crack tip is shown (indicated as "calculated"). This data was used to calculate the benefit in life time and the crack growth velocity (Fig.4b). Details can be found in $[5,6]$. It can be seen that the crack stopped in front of the grain boundary for several thousand cycles. The benefit in lifetime was calculated to 30,000 cycles. 


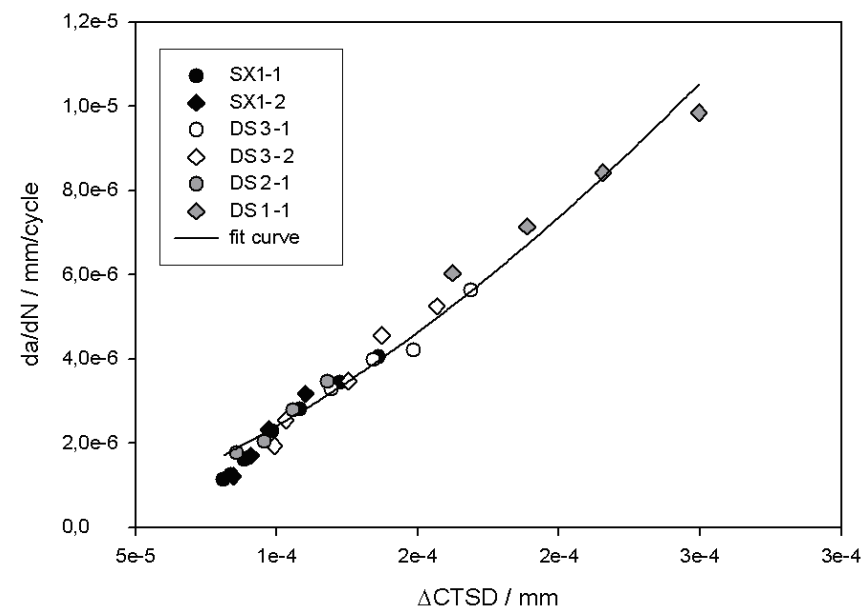

Fig.3: Crack propagation rate and crack tip sliding displacement of six different specimens.
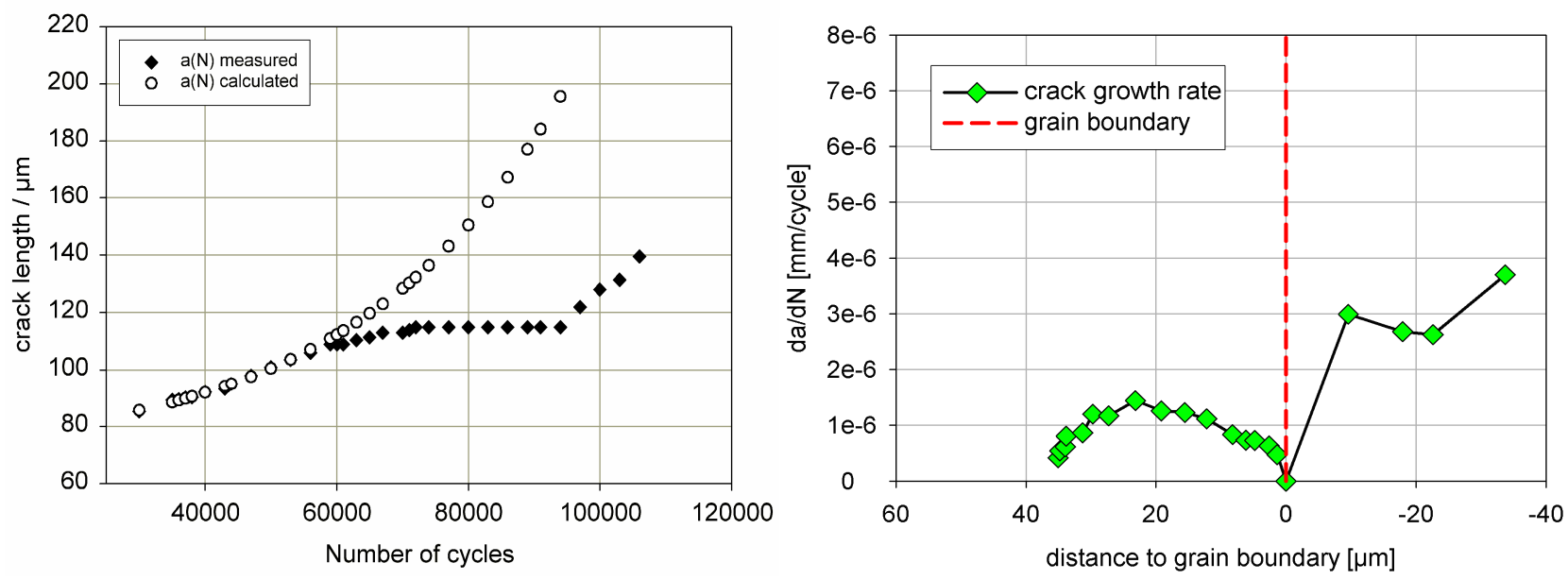

Fig.4 a) Crack length as function of the number of cycles as measured and as calculated for a specimen without grain boundary.

b) Crack propagation rate as function of the distance to the grain boundary for the crack of figure a). For further details see [5].

Influence of the distance between crack tip and grain boundary. Fatigue crack growth in stage I could be observed at two notches with different initial distance to the same grain boundary in a bicrystal. The figures $5 \mathrm{a}$ and $5 \mathrm{~b}$ show backscatter images of both notches after 30,000 cycles. The crack initiated at the notch further away from the grain boundary is much longer. It has to be pointed out that for the notch positioned closer to the grain boundary the crack arrested over a period of 7,500 cycles at the grain boundary until the crack overcame the grain boundary. In contrast, no crack arrest was observed for the notch with the higher distance to the boundary. The crack length in respect to the number of load cycles for both cracks was used to calculate the crack growth velocity by a 5-point polynominal fit. If only the velocity of the crack tip spreading towards the grain boundary is considered, the results (Fig.6) show a strong deceleration of the shorter crack which initiated closer to the boundary. For the other crack, only a slight deceleration can bee seen. After the shorter crack passed the grain boundary, both cracks should show the same velocity. However no further crack advance for the shorter crack could be found after passing the boundary. This is due to the fact that only two replica images of the crack could be made since it passed the boundary. The experiment was stopped afterwards since the other crack was too long so that further loading of the sample could lead to failure. 

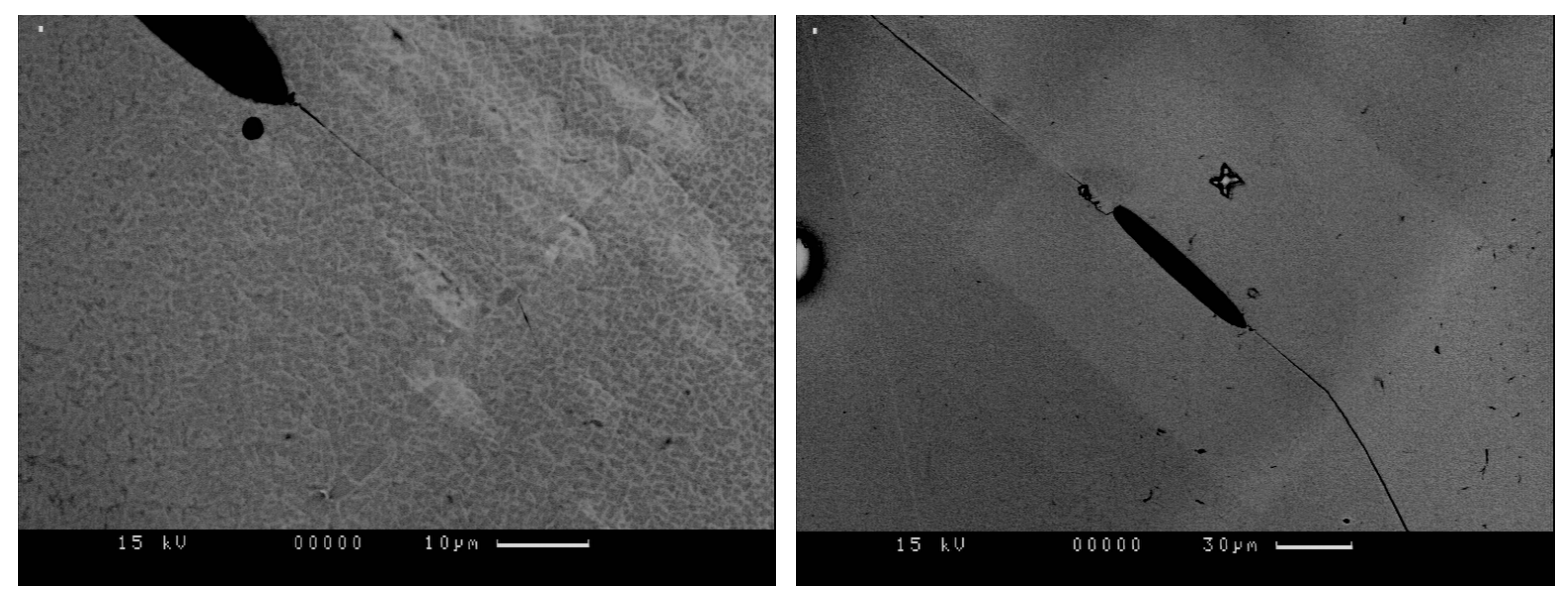

Fig.5: Notches with a distance of $30 \mu \mathrm{m}$ (left) and $50 \mu \mathrm{m}$ (right) to the grain boundary respectively.

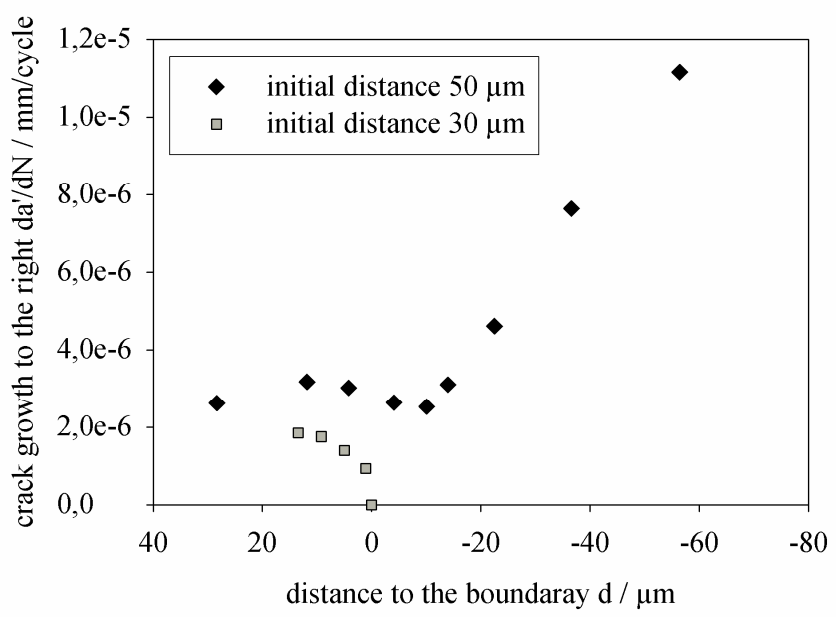

Fig.6: Crack advance for the part of the crack facing the boundary related to the distance

The observations of the two cracks are in good agreement with the theories based on continuous distributed dislocations [2,3], which was already applied in literature [8], since crack growth is more pronounced for the shorter crack when it approached the grain boundaries and both cracks accelerate after passing the boundary. It has to be pointed out, that both cracks approach the same boundary in the same orientation even in the same sample.

3D investigation of the interaction by FIB tomography. How can the resistance of a grain boundary against crack propagation be quantified? For a detailed view the problem must be examined in three dimensions. The crack has to change its propagation direction in the surface plane and perpendicular to the surface. Therefore several steps must be created at the grain boundary. The surface energy is discussed as a measure for the resistance of a grain boundary against crack propagation because the necessary energy is proportional to the additional crack surface resulting from these steps [9]. The three dimensional geometry of the cracks and the grain boundaries can be reconstructed by FIB tomography (Fig.7). Details can be found in [6, 10]. However, in this case the reduction of the crack propagation rate near the grain boundary was not due to the inclination angle between the slip systems but due to the precipitates which had to be overcome by producing additional surface steps which is nearly the same effect as described above. 

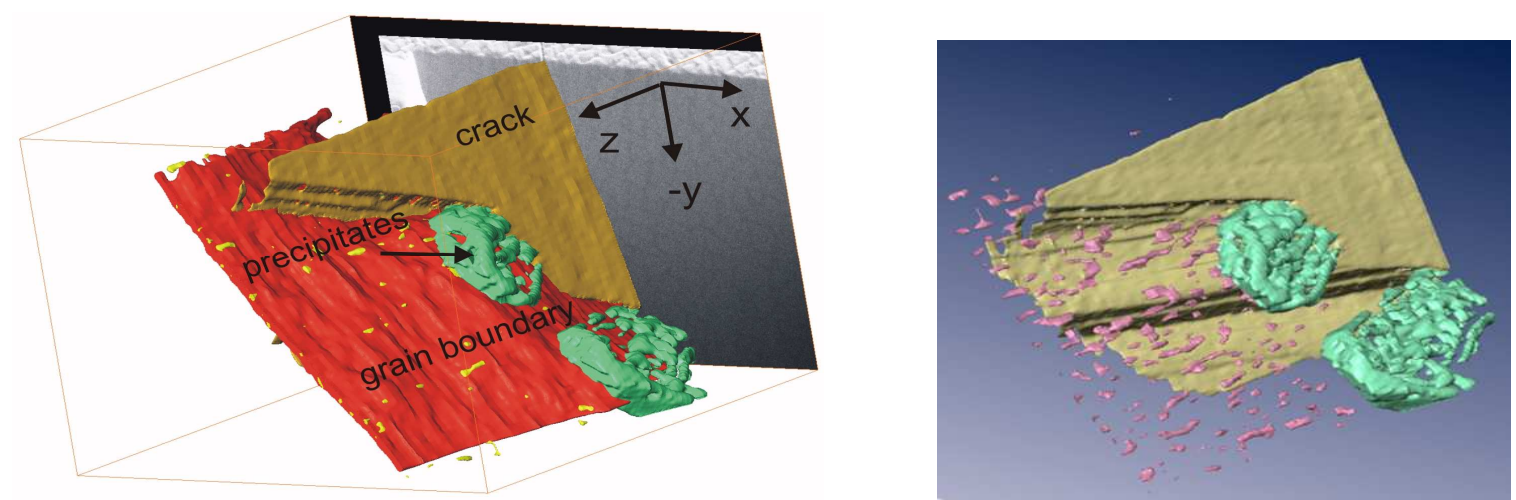

Fig.7: a) 3D-reconstruction of a crack plane and a grain boundary decorated with precipitates.

b) The grain boundary is removed and the steps on the crack plane are visible.

\section{Conclusions}

It has been shown that the material parameters for crack growth and the influence of grain boundaries on the propagation of short fatigue cracks can be investigated in detail by artificial crack initiation by FIB. Additionally, there is a sophisticated experimental set up available to investigate parameters which influence the interaction of short fatigue cracks with microstructural obstacles. Combining these techniques with local in-situ investigations of the CTSD will be helpful to improve the models for the crack propagation by replacing material parameters by general parameters based on the microstructure to avoid at least some complex and expensive fatigue tests by mechanism based fundamental models.

The support of this work by the Deutsche Forschungsgemeinschaft (DFG) is gratefully acknowledged.

\section{References}

[1] B.A. Bilby, A.H. Cotrell, K.H. Swinden, The spread of plastic yield from a notch, Proc. R. Soc. London Sect. A, 272 (1963) 304-314

[2] K. Tanaka, Y. Akiniwa, Y. Nakai, R.P. Wei, Modelling of small fatigue crack growth interacting with grain boundary, Eng. Fracture Mech., 24 (1986) 803

[3] A. Navarro, E.R. De Los Rios, Short and long Fatigue crack-growth - a unified model, Phil. Mag. A, 57 (1988) 15

[4] G.P. Potirniche, S.R. Daniewicz, J.C. Newman, Simulating small crack growth behaviour using crystal plasticity theory and finite element analysis, Fatigue \& Fract. Eng. Mater. Struct. 27 (2004) 59-71.

[5] M. Marx, W. Schaef, M. Welsch H. Vehoff, Local investigations of the interaction of microcracks with grain boundaries to quantify the qualitative models, J. of ASTM Int. 5 (9), 2008.

[6] M. Marx, W. Schäf, H. Vehoff, C. Holzapfel, Interaction of microcracks with selected interfaces: Focused ion beam for a systematic crack initiation, Mater. Sci. Eng. A 435-436 (2006) 595

[7] M.T. Welsch, M. Henning, M. Marx, H. Vehoff, Measuring the plastic zone size by orientation gradient mapping (OGM) and electron channeling contrast imaging, Adv. Eng. Mater., 9 (2007) 31-37

[8] O. Dueber, B. Kuenkler, U. Krupp, H.J. Christ, C.P. Fritzen, Int. J. Fatigue, 28 (2006) 983

[9] T. Zhai, X.P. Jiang, X. J. Li, M.D. Garratt, G.H. Bray, The grain boundary geometry for optimum resistance to growth of short fatigue cracks in high strength Al-alloys, Int $\mathrm{J}$ Fatigue, $27(2005) 1202$

[10] C. Holzapfel, W. Schäf, M. Marx, H. Vehoff, F. Mücklich: Interaction of cracks with precipitates and grain boundaries: Understanding crack growth mechanisms through FIB tomography, Scripta Mater., 56 (2007) 697-700 